\title{
Bronchioloalveolar carcinoma presenting as a thin walled cavity in a young man
}

\author{
PETRA M DE JONG, DIK L T BUSSCHER, WIM BAKKER
}

From the Departments of Pulmonology and Diagnostic Radiology, University Hospital, Leiden, The Netherlands $\overrightarrow{.}$

ABSTRACT A bronchioloalveolar carcinoma presented as a large cavitatory lesion with an irregular lining in a 28 year old man.

Bronchioloalveolar carcinoma is a malignancy that may appear as a radiologically well circumscribed, solitary peripheral nodule, in which cavitation is extremely rare. The clinical and pathological features of a cavitating bronchioloalveolar carcinoma in a young man are discussed.

\section{Case report}

A 28 year old man was admitted for evaluation of acute right sided pleuritic chest pain. He denied cough, dyspnoea, anorexia, loss of weight, or fever. No recent journeys abroad had been made. He had been smoking 20 cigarettes a day for 10 years. Because of haemoptysis a chest radiograph had been taken at another institution one year previously. This showed a sharply circumscribed, thin walled cavitary lesion $(4.5-5 \mathrm{~cm}$ in diameter) in the right lower lobe (fig 1). No further action was taken at that time and the haemoptysis did not recur. Other previous chest radiographs were not available.

At presentation we saw a young man in apparent good health. Physical examination indicated nothing abnormal apart from a right basal pleural rub. Routine laboratory investigations showed no abnormalities except for increased serum lactate dehydrogenase activity (397 (normal $<160) \mathrm{U} / \mathrm{l}$ ). Notably the erythrocyte sedimentation rate, white blood cell count, and total eosinophil count were normal. He was tuberculin negative and had negative sputum cultures. A chest radiograph and tomogram showed that the cavitary lesion in the posterobasal segment of the right lower lobe had grown. The originally smooth inner lining of the lesion now showed an irregular, nodular contour. An aortogram showed no evidence of lung sequestration. Bronchoscopy showed no abnormalities, and bronchial washings did not disclose acid fast bacilli or malignant cells.

Over the next few days a high fever $\left(39 \cdot 5^{\circ} \mathrm{C}\right)$ developed and the patient started to cough, producing purulent sputum that in culture yielded Staphylococcus aureus. On the chest radiograph the cavity was partly filled with fluid (fig 2). The patient was treated with flucloxacillin for 10 days and underwent thoracotomy once the fever had subsided.

Address for reprint requests: Dr $P$ M de Jong, Department of Pulmonology, University Hospital, Rynsburgerweg 10, 2333 AA Leiden, The Netherlands.
At thoracotomy a smooth surfaced and partly necrotic $\overrightarrow{\vec{F}}$ tumour mass $(11 \mathrm{~cm}$ in diameter) was found, fixed to the $x$ diaphragm. Lobectomy with total removal of the tumour was $\rightarrow$ performed. Microscopically this was seen to be a bron- $\omega$ chioloalveolar carcinoma. Lymph nodes were not affected.

\section{Discuscion}

Bronchioloalveolar carcinoma is known as a malignancy $\bar{z}$ with an insidious onset. ${ }^{1}$ It accounts for $1.5-6.5 \%$ of primary lung carcinomas and commonly appears as a localised, well $\stackrel{\rho}{\supset}$ circumscribed, solitary peripheral nodule in the lung paren- $\vec{c}$ chyma. ${ }^{23}$ Cavitation must be very rare, for in several large 0 series with a total of 358 patients this radiographic. appearance was never mentioned, ${ }^{1-4}$ though a recent textbook states without further reference that cavitation may occur in a large lesion. ${ }^{5}$ Apart from the localised form of bronchioloalveolar carcinoma a diffuse pattern has been des-

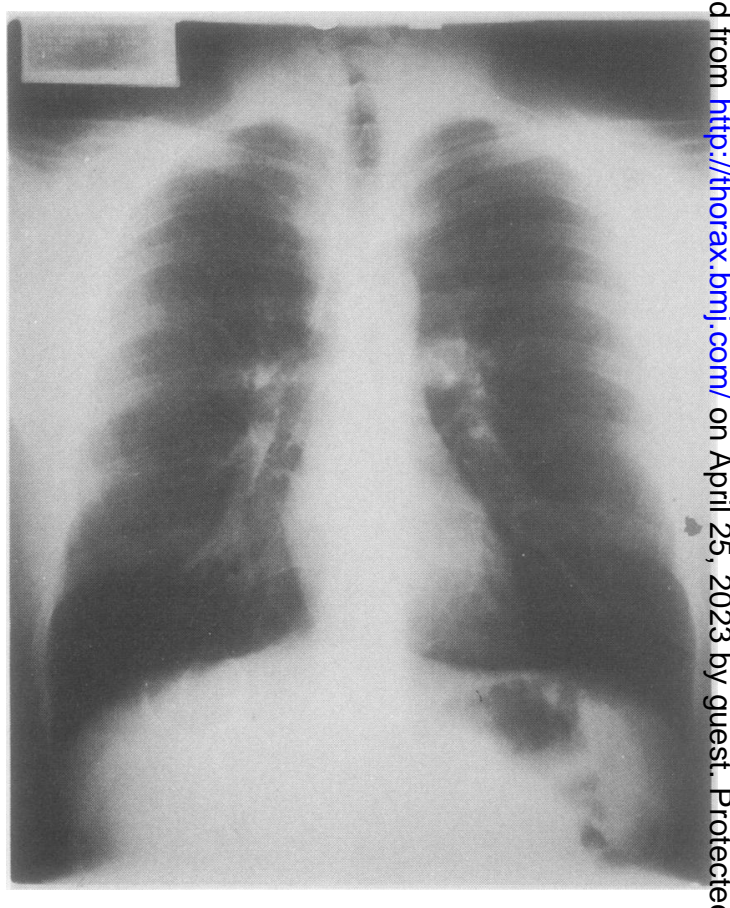

Fig 1 Cavitary lesion in the right lower lobe one year before the present admission. 


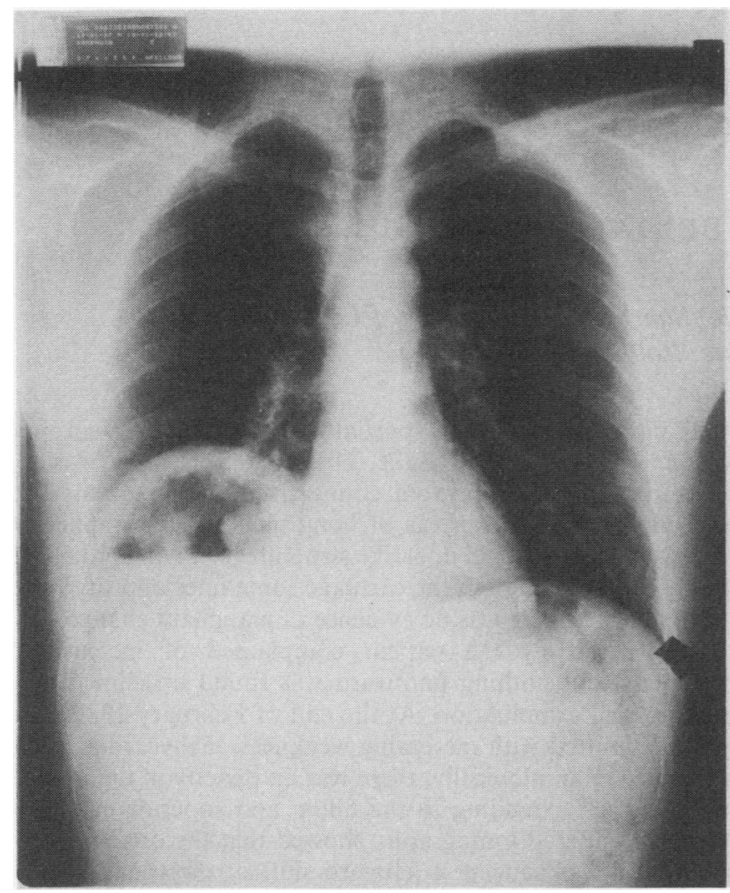

Fig 2 The cavitary lesion one year later: it is larger than in figure 1 and now shows a fluid level.

cribed. Miller et al believe that these different patterns represent one histological tumour type with two distinct clinical patterns of behaviour. ${ }^{2}$ Gender and smoking history do not appear to be related to the tumour. The disease generally occurs in the elderly. Patients as young as ours are extremely rare, although a case in a 15 year old boy has been reported. ${ }^{6}$ The age range in one series of 41 patients was 37-83 years.

The term bronchioloalveolar carcinoma is confined to tumours originating in bronchioles or alveoli.' Microscopically, the tumour cells line the alveolar septa, the normal architecture of the lung acting as a supporting stroma. Larger bronchi are not affected. ${ }^{8}$ Obliteration of alveoli with tumour tissue often occurs in the centre of the lesion but not at the periphery. The malignant cell population varies greatly, ranging from well differentiated and mucin producing to poorly differentiated cells of hobnail appearance. ${ }^{9}$ The diffuse form almost invariably leads to death within three years irrespective of treatment. The localised form on the other hand has a five year survival rate of over $60 \%$ after surgical removal. 910

Radiological features of a cavity suggesting malignancy include multiple holes; a nodular, ill defined inner or outer wall; and eccentric excavation. "On the other hand, a well defined smooth, thin walled, air containing lesion, which our patient had one year before admission (fig 1), suggests a benign lesion. The radiographic pattern seen in figure 2 , with an irregular, nodular lining of the cavitary lesion, was considered to be very suggestive of malignancy. In retrospect, we cannot be sure whether the original cyst like appearance on the chest radiograph represented a malignant tumour from the onset or a benign cyst in which a carcinoma developed; but the latter seems unlikely.

This case clearly shows the importance of awareness that a cavitating lesion may be malignant even when the patient is young and the lesion is thin walled and sharply outlined.

We gratefully acknowledge the valuable comments of Professor E Scheffer, Department of Pathology, University Hospital, Leiden.

\section{References}

1 Hill CA. Bronchioloalveolar carcinoma: a review. Radiology 1984;150:15-20.

2 Miller WT, Husted J, Freiman D, Atkinson B, Pietra GC. Bronchioloalveolar carcinoma: two clinical entities with one pathologic diagnosis. AJR 1978;130:905-12.

3 Epstein DM, Gefter WB, Miller WT. Lobar bronchioloalveolar cell carcinoma. AJR 1982;139:463-8.

4 Story CF, Knudson KP, Lawrence BJ. Bronchial (alveolar cell) carcinoma of the lung. $J$ Thorac Surg 1953;26:331-403.

5 Thurlbeck WM. Pathology of the lung. New York: Thieme, 1988:352.

6 Anonymous. Case records of the Massachusetts General Hospital, No 4. N Engl J Med 1976;294:210-7.

7 Manning JT, Spurt HJ, Tschen JA. Bronchioloalveolar carcinoma. Cancer 1984;54:525-34.

8 Carter D, Eggelston JC. Atlas of tumor pathology. Second series, fascicle 17. Washington DC: Armed Forces Institute of Pathology, 1980:127-47.

9 Schraufnagel D, Peloquin A, Pare JA, Wang NS. Differentiating bronchioloalveolar carcinoma. Am Rev Respir Dis 1982; 125:74-9.

10 Clayton F. Bronchioloalveolar carcinoma. Cancer 1986;57: $1555-64$.

11 Felson B. Chest roentgenology. Philadelphia: Saunders, 1973:319. 